\title{
Mathematical Analysis of the Pharmacokinetic-Pharmacodynamic (PKPD) Behaviour of Monoclonal Antibodies: Predicting in vivo Potency
}

\author{
Philip J. Aston ${ }^{\mathrm{a}, *}$, Gianne Derks ${ }^{\mathrm{a}}$, Adewale Raji ${ }^{\mathrm{b}, \mathrm{a}, \mathrm{c}}$, Balaji M. Agoram ${ }^{\mathrm{b}, 1}$, Piet H. van der \\ Graaf $^{b}$ \\ ${ }^{a}$ Department of Mathematics, University of Surrey, Guildford GU2 7XH, UK \\ ${ }^{b}$ Pfizer, PDM, Clinical Pharmacology and Pharmacometrics, Sandwich CT13 9NJ, UK \\ ${ }^{c}$ Knowledge Transfer Centre, University of Reading, Reading RG6 6AH, UK
}

\begin{abstract}
We consider the relationship between the target affinity of a monoclonal antibody and its in vivo potency. The dynamics of the system is described mathematically by a target-mediated drug disposition model. As a measure of potency, we consider the minimum level of the free receptor following a single bolus injection of the ligand into the plasma compartment. From the differential equations, we derive two expressions for this minimum level in terms of the parameters of the problem, one of which is valid over the full range of values of the equilibrium dissociation constant $K_{\mathrm{D}}$ and the other which is valid only for a large drug dose or for a small value of $K_{\mathrm{D}}$. Both of these formulae show that the potency achieved by increasing the association constant $k_{\text {on }}$ can be very different from the potency achieved by decreasing the dissociation constant $k_{\text {off }}$. In particular, there is a saturation effect when decreasing $k_{\text {off }}$ where the increase in potency that can be achieved is limited, whereas there is no such effect when increasing $k_{\text {on }}$. Thus, for certain monoclonal antibodies, an increase in potency may be better achieved by increasing $k_{\text {on }}$ than by decreasing $k_{\text {off }}$.
\end{abstract}

Keywords: Pharmacology, efficacy, affinity, target-mediated drug disposition, IgE.

\section{Introduction}

Since their introduction as clinical agents in the 1980s, monoclonal antibodies (mAbs) have become one of the fastest growing classes of therapeutic modalities with $24 \mathrm{mAbs}$ currently on the market in the US [1] and more than 200 in clinical trials. The increasing interest in biologicals in general, and $\mathrm{mAbs}$ in particular, is partly due to their high success rate during preclinical and clinical development [1]. It has been suggested that an important element that has contributed to this success is the fact that, compared to small molecules, the pharmacokinetic-pharmacodynamic (PKPD) properties of mAbs are more amenable to quantitative, mechanistic modelling and simulation (M\&S)-based translation approaches across preclinical and clinical research $[2,3,4,5]$. A specific example of this is the implementation of the principles of target-mediated drug disposition (TMDD) into quantitative pharmacological models to describe and predict PKPD behaviour of mAbs. Levy [6] introduced the term TMDD to describe the observations that for certain potent and selective drugs the fraction (relative to the dose) bound to the pharmacological target may be so high that it influences their disposition, i.e. PK and PD become interdependent. Mager and Jusko [7] were the first to propose a general PK model for drugs exhibiting TMDD, which has provided the basis for extensive further studies and development (see for example $[8,9,10,11,5]$ ).

\footnotetext{
*Corresponding author. Tel: +44 1483 682631; fax: +44 1483686071.

Email addresses: P.Aston@surrey.ac.uk (Philip J. Aston), G.Derks@surrey.ac.uk (Gianne Derks), AgoramB@MedImmune.com (Balaji M. Agoram), Piet.Van.Der.Graaf@pfizer.com (Piet H. van der Graaf)

${ }^{1}$ Present address: MedImmune, Pharmacokinetics/Dynamics and Bioanalysis, Cambridge CB21 6GH, UK
} 


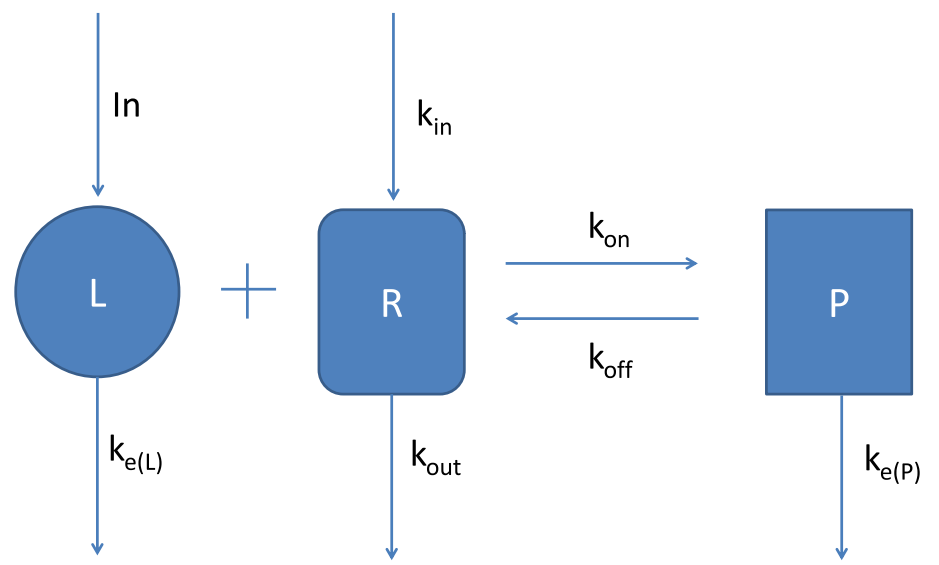

Figure 1: The TMDD reaction mechanism

Experimental confirmation of TMDD model predictions was provided in a recent elegant study by Abraham et al. [12], who showed marked differences in interferon (INF)- $\beta$ PK in wild-type and type-1 INF $\alpha / \beta$ receptor knockout mice.

Although originally proposed to describe the effects of extensive drug-target binding in tissues, TMDD has received most interest as a saturable clearance mechanism (due to, for example, receptor-mediated endocytosis; see [13]) for biologics, specifically mAbs. One specific example of the impact of mechanism-based PKPD models based on TMDD principles in drug discovery and development comes from the area of anti-IgE mAbs. The first generation molecule, omalizumab (Xolair $\AA$ [14]) is used for the treatment of allergic asthma and there has been an interest to develop more potent, second-generation mAbs with improved clinical efficacy profile $[2,4]$. Specifically, TMDD models have been used to explore the impact of mAb affinity for IgE in relation to in vivo potency (the dose required for a given clinical effect). Through a sensitivity analysis, Agoram et al. [2] predicted that a ten-fold increase in mAb affinity for IgE would result in an approximately two-fold reduction in dose compared to omalizumab but that further increases in affinity were not predicted to result in additional potency improvements. In contrast, simulations did suggest that increased on-rate of $\mathrm{mAb}$ binding to IgE could have additional impact on the in vivo potency [2]. However no formal analysis was presented to underwrite this hypothesis. Similarly, Sarkar et al. [15] demonstrated the use of mechanistic models for the design of optimal biologic therapeutics such as GCSF. Therefore, some examples exist in the literature on the exploration of the affinity-potency relationships using detailed mathematical models of cellular processes, but these analyses are heuristic in nature. A systematic analysis of how mechanistic TMDD models can be employed in potency estimation of candidates is not yet available. Inspired by the beforementioned case studies and recent examples of how a rigorous mathematical analysis can aid our understanding of complex pharmacological systems and provide tools to predict essential PKPD properties of mAbs $[16,10,13]$ we explore the behaviour of a TMDD model with respect to the relationship between the target affinity of the $\mathrm{mAb}$ and its in vivo potency.

Section 1 gives the background for this study and section 2 describes the model development from the reaction mechanism. The mathematical analysis of the resulting kinetic rate equations is depicted in this section for easy manipulation and understanding of the model. In sections 3 and 4, the results and discussion of the model dynamics are presented and mathematical expressions to approximate the potency of the drug are derived here. Section 5 gives a further analysis and validation of the relationships derived in the previous sections. Finally, the conclusions and recommendations arising from these studies are given in section 6 . 


\section{Model Equations}

In this study, a one-compartment model based on the original work of Levy [6] will be employed. In this investigation, the ligand $L$ (drug) binds reversibly with the receptor $R$ to form a receptorligand complex $P$ as shown in the scheme in Fig. 1. The TMDD model assumes a mechanism-based reaction to explain the drug-receptor interaction. The parameters of the model are the binding rate constants $k_{\text {on }}$ and $k_{\text {off }}$, the receptor turnover and elimination rates $k_{\text {in }}$ and $k_{\text {out }}$, and the elimination rates of the ligand and complex $k_{\mathrm{e}(\mathrm{L})}$ and $k_{\mathrm{e}(\mathrm{P})}$. The system is assumed to be initially at steady state, into which a single bolus infusion $L_{0}$ of the ligand into the central (plasma) compartment is made (represented in Fig. 1 by 'In').

From the mechanism of the TMDD reaction shown in Fig. 1 the mathematical model can be derived using the Law of Mass Action giving the differential equations

$$
\begin{aligned}
\frac{d L}{d t} & =-k_{\mathrm{e}(\mathrm{L})} L-k_{\mathrm{on}} L R+k_{\mathrm{off}} P \\
\frac{d R}{d t} & =k_{\mathrm{in}}-k_{\mathrm{out}} R-k_{\mathrm{on}} L R+k_{\mathrm{off}} P \\
\frac{d P}{d t} & =k_{\mathrm{on}} L R-k_{\mathrm{off}} P-k_{\mathrm{e}(\mathrm{P})} P
\end{aligned}
$$

The steady state of this system is given by $L=P=0, R=k_{\text {in }} / k_{\text {out }}$. Adding the bolus injection gives the initial conditions

$$
L(0)=L_{0}, \quad R(0)=R_{0}=\frac{k_{\text {in }}}{k_{\text {out }}}, \quad P(0)=0 .
$$

We non-dimensionalise these equations by defining the dimensionless variables

$$
x=\frac{L}{L_{0}}, \quad y=\frac{R}{R_{0}}, \quad z=\frac{P}{R_{0}}, \quad \tau=k_{\text {out }} t .
$$

We also define the non-dimensional parameter

$$
\mu=\frac{R_{0}}{L_{0}} .
$$

This non-dimensionalisation is different from the one used by Peletier and Gabrielsson [10] who defined the new time variable as $\tau=k_{\mathrm{on}} R_{0} t$. The reason for this is that later on we want to explore the limits of $k_{\mathrm{on}} \rightarrow 0$ and $k_{\mathrm{on}} \rightarrow \infty$, and so we do not want $k_{\mathrm{on}}$ to be used in the rescaling of time. We also note that $k_{\text {out }}$ must be non-zero for the steady state $R_{0}$ to exist, and so this is an obvious choice of parameter to use for the non-dimensionalisation. In terms of these non-dimensional quantities, equations (1)-(3) become

$$
\begin{aligned}
& \frac{d x}{d \tau}=-K_{1} x-K_{2} x y+\mu K_{3} z \\
& \frac{d y}{d \tau}=1-y-\frac{K_{2}}{\mu} x y+K_{3} z \\
& \frac{d z}{d \tau}=\frac{K_{2}}{\mu} x y-\left(K_{3}+K_{4}\right) z
\end{aligned}
$$

with initial conditions

$$
x(0)=1, \quad y(0)=1, \quad z(0)=0,
$$

where the dimensionless parameters are defined by

$$
K_{1}=\frac{k_{\mathrm{e}(\mathrm{L})}}{k_{\text {out }}}, \quad K_{2}=\frac{k_{\text {on }} R_{0}}{k_{\text {out }}}, \quad K_{3}=\frac{k_{\text {off }}}{k_{\text {out }}}, \quad K_{4}=\frac{k_{\mathrm{e}(\mathrm{P})}}{k_{\text {out }}} .
$$

The constant term in (7) is derived by using the definition of the steady state $R_{0}$ in (4). We note that the six parameters and one initial value $\left(L_{0}\right)$ of the dimensional equations have been reduced 


\begin{tabular}{cccc}
\hline Kinetic Constant & Value & Parameter & Value \\
\hline$k_{\mathrm{e}(\mathrm{L})}$ & $0.024 \mathrm{day}^{-1}$ & $K_{1}$ & 0.02916 \\
$k_{\mathrm{e}(\mathrm{P})}$ & $0.201 \mathrm{day}^{-1}$ & $K_{2}$ & 1.93353 \\
$k_{\text {out }}$ & $0.823 \mathrm{day}^{-1}$ & $K_{3}$ & 1.09356 \\
$k_{\text {off }}$ & $0.900 \mathrm{day}^{-1}$ & $K_{4}$ & 0.24423 \\
$k_{\text {on }}$ & $0.592(\mathrm{nM} \mathrm{day})^{-1}$ & $\mu$ & 0.18144 \\
$K_{D}$ & $1.520 \mathrm{nM}$ & & \\
$k_{\text {in }}$ & $k_{\text {out }} R_{0} \mathrm{nM} \mathrm{day}$ & & \\
$L_{0}$ & $14.8148 \mathrm{nM}$ & & \\
$R_{0}$ & $2.688 \mathrm{nM}$ & &
\end{tabular}

Table 1: Numerical values of the dimensioned and dimensionless constants for IgE mAb omalizumab [2].

to five non-dimensional constants for the non-dimensional equations, which simplifies the model equations while keeping the essence of the system.

We use parameter values from the $\operatorname{IgE~mAb~omalizumab~case~study~[2]~as~an~example.~The~}$ numerical values of the dimensioned and dimensionless parameters for this case are given in Table 1 , where we have also included the value of the equilibrium dissociation constant $K_{D}=k_{\text {off }} / k_{\text {on }}$. For the dimensioned parameters, we use these units hereafter without mentioning them specifically. Using these parameters, the time profile of the ligand, receptor and ligand-receptor complex are shown in Fig. 2 for a short time (just past the minimum of $R$ ) and for a longer time interval in Fig. 3. Clearly, plots of the non-dimensional quantities $x, y$ and $z$ will be similar but with a difference scale on the vertical axis.

These numerical results show different phases occurring. Initially, in a very short time, the receptor $R$ drops down to a low value while the product $P$ shows a corresponding jump. There is also an initial sharp but small drop in the ligand $L$. This phase is dominated by the binding action of the ligand to the receptor resulting in the product. In the next phase, which happens on a much slower timescale, the receptor and the product gradually increase, while the ligand continues with a gradual decrease. Finally, the system settles back to its steady state values.

\section{Approximation of the Drug Potency - Method 1}

In our analysis, the in vivo potency of the drug is defined to be the minimum free receptor level that can be achieved for a particular dose of the drug. Mathematically, this minimum receptor level, $R_{\text {min }}$, is obtained by finding the minimum point on the receptor-time profile (see Fig. 2(b)) and is therefore defined as the point where the time derivative of $R$ (or $y$ in dimensionless form) vanishes.

Setting $\dot{y}=0$ in (7) implies that this minimum occurs when

$$
1-y-\frac{K_{2}}{\mu} x y+K_{3} z=0,
$$

which gives

$$
y=\frac{\mu\left(1+K_{3} z\right)}{\mu+K_{2} x} .
$$

The problem with this is that we do not know the values of $x$ and $z$ when the minimum occurs, and so all this gives us is a two-dimensional surface in the three-dimensional phase space on which the minimum must occur but the position on this surface where the actual minimum occurs for the given trajectory is not known.

To address this problem, we rewrite equations (6)-(8) in terms of different variables. In particular, we introduce a non-dimensional form of the total amount of ligand (free and bound) together 

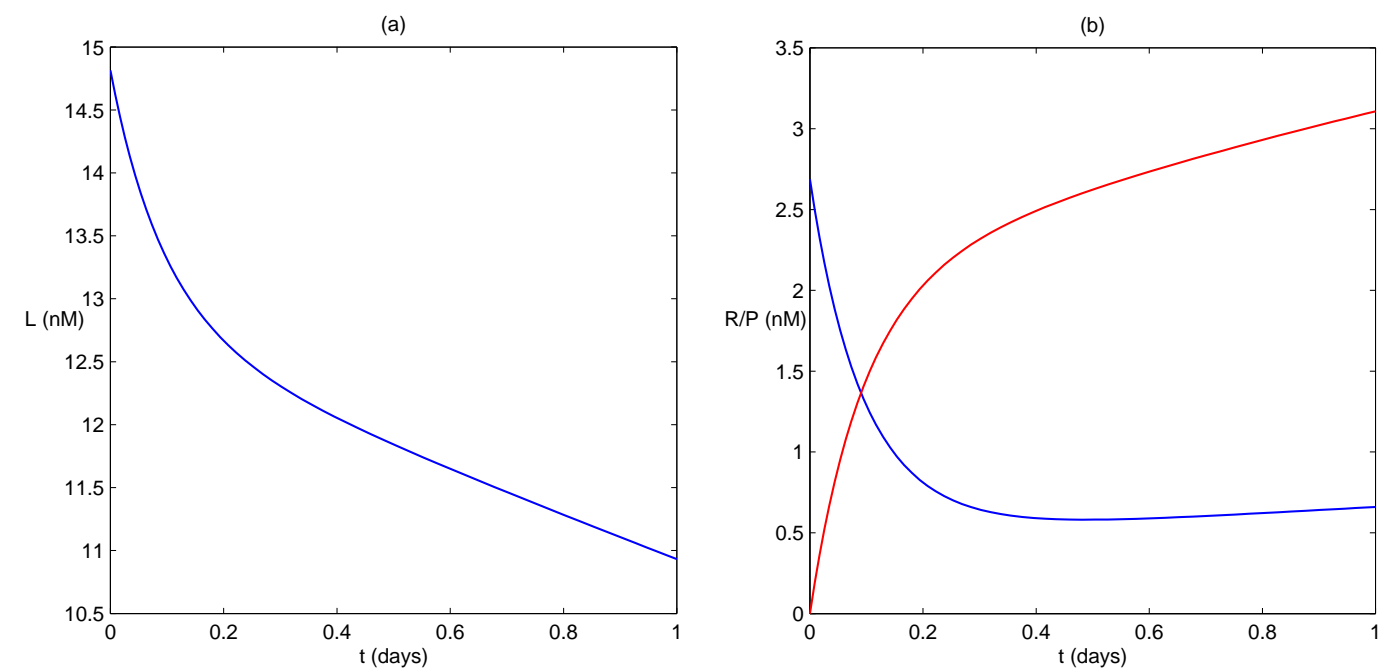

Figure 2: Concentration-time profile for $0 \leq t \leq 1$ of (a) the free ligand $L$, and (b) the free receptor $R$ (blue) and the ligand-receptor complex $P$ (red).
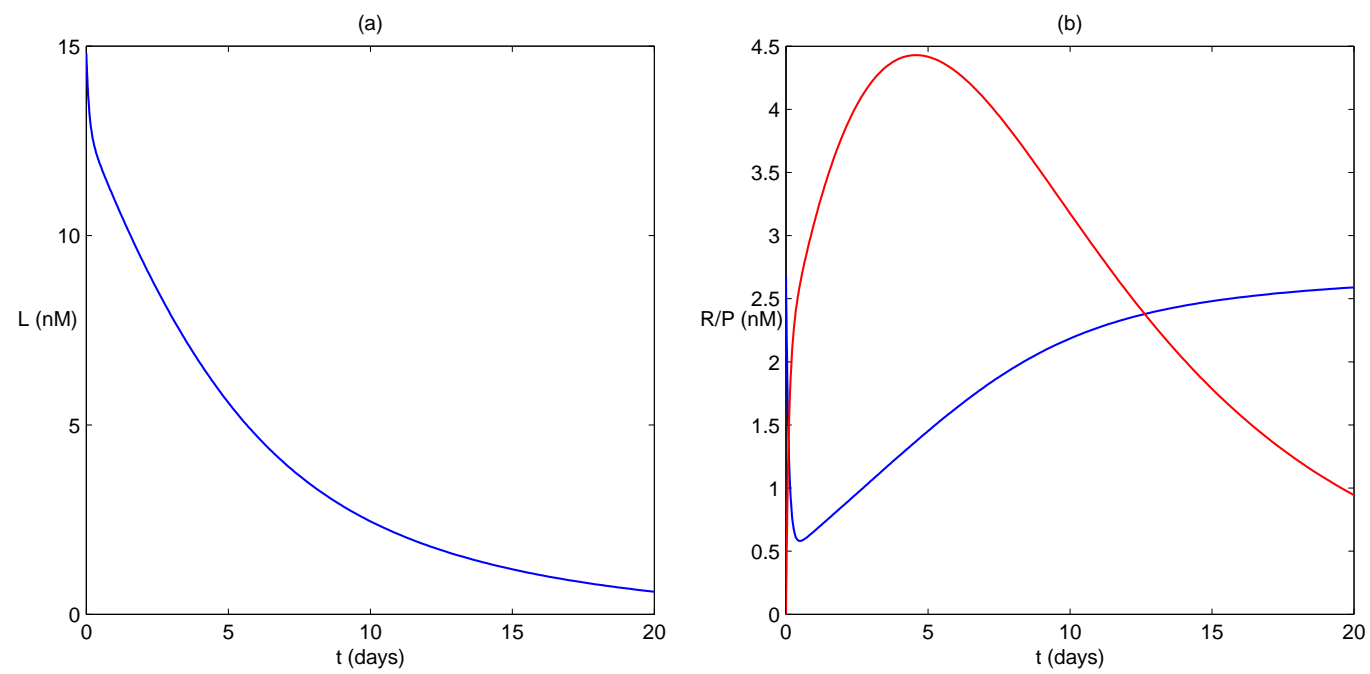

Figure 3: Concentration-time profile for $0 \leq t \leq 20$ of (a) the free ligand $L$, and (b) the free receptor $R$ (blue) and the ligand-receptor complex $P$ (red).
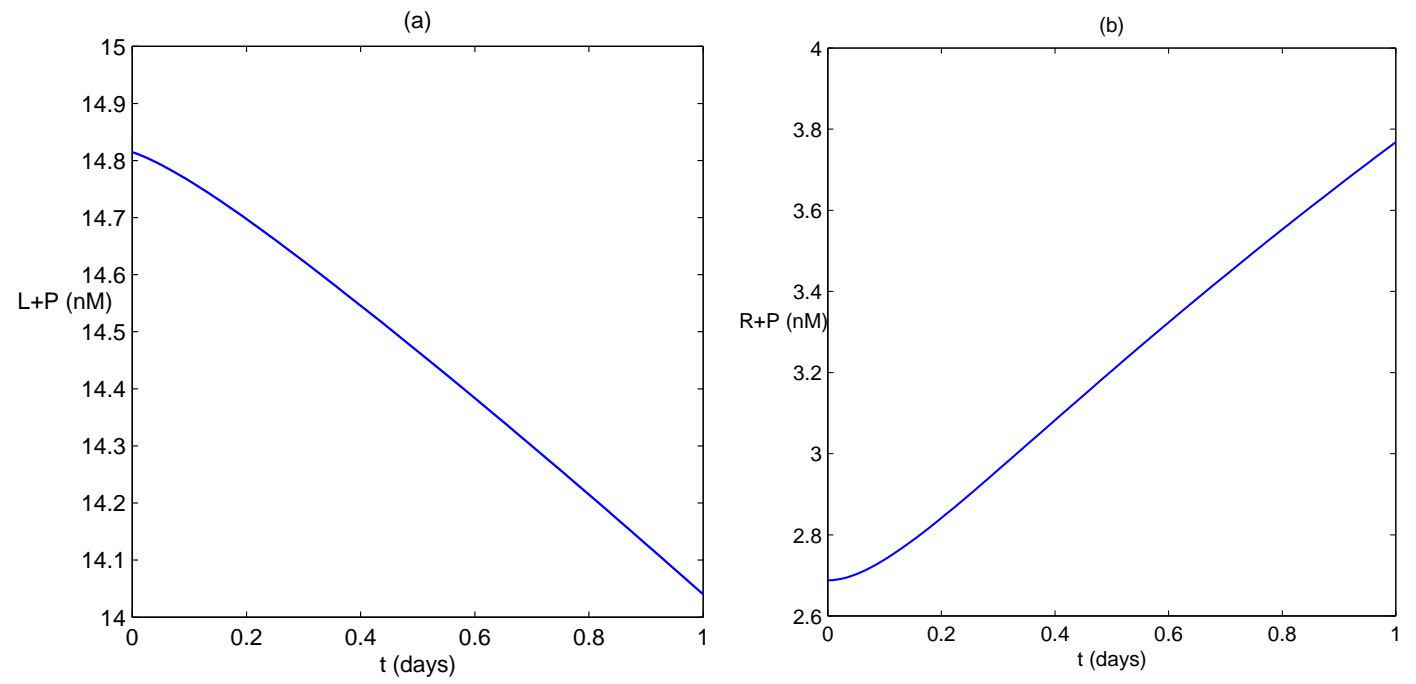

Figure 4: Concentration-time profile of (a) the total ligand $L+P$ and (b) the total receptor $R+P$. Note the small variation in values on the vertical $L+P$-axis. 
with the total amount of receptor (free and bound). Thus, we define

$$
\begin{aligned}
& u=\frac{L+P}{L_{0}}=x+\mu z \\
& v=\frac{R+P}{R_{0}}=y+z
\end{aligned}
$$

We now rewrite our equations in terms of the variables $u, v$ and $y$, giving

$$
\begin{aligned}
\dot{u} & =-K_{1} u-\mu\left(K_{4}-K_{1}\right)(v-y) \\
\dot{v} & =1-y-K_{4}(v-y) \\
\dot{y} & =1-y-\frac{K_{2}}{\mu} u y+\left(K_{2} y+K_{3}\right)(v-y)
\end{aligned}
$$

with initial conditions

$$
u(0)=1, \quad v(0)=1, \quad y(0)=1 .
$$

The concentration-time profile for the total ligand $L+P$ and the total receptor $R+P$ are shown in Fig. 4.

We assume that the amount $L_{0}$ of ligand injected is greater than the steady state value of the receptor $R_{0}$ so that the parameter $\mu$, which is defined in (5), satisfies $\mu \ll 1$.

As before, we can set $\dot{y}=0$ in (11) giving

$$
1-y-\frac{K_{2}}{\mu} u y+\left(K_{2} y+K_{3}\right)(v-y)=0 .
$$

We note that in this case, we have a quadratic equation for $y$ which has one positive and one negative solution. The positive solution is given by

$$
y=-\frac{1}{2 \mu K_{2}}\left(\left[K_{2} u+\mu\left(1+K_{3}-K_{2} v\right)\right]-\sqrt{\left[\left(K_{2} u+\mu\left(1+K_{3}-K_{2} v\right)\right)^{2}+4 \mu^{2} K_{2}\left(1+K_{3} v\right]\right.}\right) .
$$

Since we have assumed that $\mu$ is small, the first term under the square root will dominate the second. Thus, taking this first term out of the square root and then expanding the square root term gives

$$
y=\frac{\mu\left(1+K_{3} v\right)}{K_{2} u+\mu\left(1+K_{3}-K_{2} v\right)}+O\left(\mu^{3}\right) .
$$

Of course, we still have the problem that this formula for $y$ involves the variables $u$ and $v$. However, the advantage of working with equations (9)-(11) is that the variables $u$ and $v$ evolve on a much slower timescale than $y$. To see this, we note that

$$
\dot{u}(0)=-K_{1}, \quad \dot{v}(0)=0, \quad \dot{y}(0)=-\frac{K_{2}}{\mu} .
$$

Provided that $\mu K_{1} / K_{2}=k_{\mathrm{e}(\mathrm{L})} /\left(k_{\mathrm{on}} L_{0}\right)$ is small, which is the case for the parameter values we are considering, then the derivative of $y$ at time zero is much greater than the corresponding derivatives of $u$ and $v$, and hence $y$ will initially change at a much faster rate than $u$ and $v$, as can be seen from Figs 2 and 4. Indeed, the variables in Fig. 4 do not show the initial sharp change that can be observed for $R$ (or equivalently $y$ ) in Fig. 2 .

During this initial fast phase, $u$ and $v$ remain approximately constant while $y$ quickly decreases to its minimum and so we can approximate this minimum by setting $u=u(0)=1$ and $v=v(0)=$ 1. Substituting these values into (12) and ignoring the higher order terms gives an approximation to the minimum value of $y$ as

$$
y_{\min }^{(1)}=\frac{\mu\left(1+K_{3}\right)}{K_{2}+\mu\left(1+K_{3}-K_{2}\right)} .
$$


Converting this expression back to the dimensional variables, we obtain an approximation for the minimum of $R$ as

$$
R_{\min }^{(1)}=\frac{R_{0}\left(k_{\text {off }}+k_{\text {out }}\right)}{k_{\text {on }}\left(L_{0}-R_{0}\right)+k_{\text {off }}+k_{\text {out }}} .
$$

Looking at the limiting cases, we see that varying $k_{\text {on }}$ gives

$$
\lim _{k_{\mathrm{on}} \rightarrow 0} R_{\min }^{(1)}=R_{0}, \quad \lim _{k_{\mathrm{on}} \rightarrow \infty} R_{\min }^{(1)}=0 .
$$

Similarly, varying $k_{\text {off }}$ gives

$$
\lim _{k_{\text {off }} \rightarrow \infty} R_{\min }^{(1)}=R_{0}, \quad \lim _{k_{\text {off }} \rightarrow 0} R_{\min }^{(1)}=\frac{R_{0} k_{\text {out }}}{k_{\text {on }}\left(L_{0}-R_{0}\right)+k_{\text {out }}},
$$

We note that in the limit as $K_{D}=k_{\mathrm{off}} / k_{\mathrm{on}} \rightarrow \infty$ there is no reaction between the ligand and the receptor and hence no product is formed. Thus, $R$ remains at its steady state value $R_{0}$ and so we have obtained the correct limit in this case.

In the alternative case when $K_{D}=k_{\text {off }} / k_{\text {on }} \rightarrow 0$, we note that increasing $k_{\text {on }}$ results in the minimum level of the receptor decreasing to zero, while decreasing $k_{\text {off }}$ results in the minimum receptor level decreasing to a non-zero level.

We note in passing that the formula for $R_{\mathrm{min}}^{(1)}$ can be expressed in terms of the single parameter $\left(k_{\text {off }}+k_{\text {out }}\right) / k_{\text {on }}$ for given $L_{0}$ and $R_{0}$ and so an increase in $k_{\text {on }}$ will have the same effect as a corresponding decrease in $k_{\text {off }}+k_{\text {out }}$. However, $k_{\text {out }}$ is not a constant over which we have any control, and so this observation is not particularly helpful in practice.

\section{Approximation of the Drug Potency - Method 2}

In the previous section, we worked with the dimensionless variables for the total ligand $(u)$, total receptor $(v)$ and free receptor $(y)$. We now consider a similar approach using instead the dimensionless variables for the total ligand $(u)$, free receptor $(y)$ and product $(z)$. In this case, the condition for the minimum of $y$ (i.e. $\dot{y}=0$ ) gives

$$
\mu(1-y)-K_{2}(u-\mu z) y+\mu K_{3} z=0,
$$

which is linear in $y$. Solving this equation for $y$ we obtain

$$
y=\frac{\mu\left(1+K_{3} z\right)}{K_{2} u+\mu\left(1-K_{2} z\right)} .
$$

As before, we note that $u$ remains approximately constant during the initial fast phase and so we again take $u=1$. However, $z$ changes rapidly during the initial phase, as does $y$, and so we cannot use the initial value of $z$. The assumption used previously that $v=1$ implies that $y+z=1$ or $z=1-y$. Now at the minimum point, if we assume that $y=O(\mu)$, then $z=1-O(\mu)$ at this point. Since $\mu$ is assumed to be small, we simply take $z=1$ as the leading order approximation. With these two assumptions, we obtain an approximation for the minimal receptor level as

$$
y_{\min }^{(2)}=\frac{\mu\left(1+K_{3}\right)}{K_{2}+\mu\left(1-K_{2}\right)},
$$

which is very similar, but not quite the same, as the previous approximation $y_{\min }^{(1)}$ given in (13). Converting back to the original coordinates gives

$$
R_{\mathrm{min}}^{(2)}=\frac{R_{0}\left(k_{\mathrm{out}}+k_{\mathrm{off}}\right)}{k_{\mathrm{on}}\left(L_{0}-R_{0}\right)+k_{\mathrm{out}}} .
$$

It is easily verified that $y_{\min }^{(2)}=y_{\min }^{(1)}+O\left(\mu^{2}\right)$. We also note that

$$
\lim _{k_{\mathrm{on}} \rightarrow \infty} R_{\min }^{(2)}=\lim _{k_{\mathrm{on}} \rightarrow \infty} R_{\min }^{(1)}=0,
$$


and that

$$
\lim _{k_{\text {off }} \rightarrow 0} R_{\text {min }}^{(2)}=\lim _{k_{\text {off }} \rightarrow 0} R_{\text {min }}^{(1)}=\frac{R_{0} k_{\text {out }}}{k_{\text {on }}\left(L_{0}-R_{0}\right)+k_{\text {out }}},
$$

and so the limiting values for the two approximations $R_{\min }^{(1)}$ and $R_{\min }^{(2)}$ as $K_{D}=k_{\text {off }} / k_{\text {on }} \rightarrow 0$ are the same. However, in the other limit with $K_{D}=k_{\text {off }} / k_{\text {on }} \rightarrow \infty$, we find that

$$
\lim _{k_{\mathrm{on}} \rightarrow 0} R_{\mathrm{min}}^{(2)}=R_{0}\left(1+\frac{k_{\mathrm{off}}}{k_{\mathrm{out}}}\right), \quad \lim _{k_{\mathrm{off}} \rightarrow \infty} R_{\mathrm{min}}^{(2)}=\infty .
$$

Thus, the correct values are not obtained in this limit. This is expected since we assumed above that $y_{\text {min }}=O(\mu)$, which in turn implies that $\left(1+K_{3}\right) / K_{2}=O(1)$. When $k_{\text {off }} / k_{\text {on }}$ is large, then $K_{3} / K_{2}$ is also large and so this condition is violated and the approximation is not valid.

The main assumption that we use in deriving (18) is that the minimum value of $y$ is small. One way that this can be achieved is to assume that $\mu=R_{0} / L_{0}$ is small. However, this is not the only condition that can give a small minimum value for $y$. The small minimum value is obtained as a consequence of the reaction between $L$ and $R$, which produces the product $P$, happening on a much faster timescale than the other dynamic processes, and from the non-dimensional equations (6)-(8), we can see that the reduction of $y$ will happen on a fast timescale if the product term $K_{2} x y / \mu$ is larger than the other terms in (7). The coefficient $K_{2} / \mu=k_{\text {on }} L_{0} / k_{\text {out }}$ will be larger than the other coefficients if $k_{\mathrm{on}} L_{0}$ is large relative to $k_{\text {out }}$ and $k_{\text {off }}$. Another requirement is that $x$ should not be depleted too quickly by elimination relative to the reaction rate, and this requires that $k_{\mathrm{e}(\mathrm{L})}$ is small relative to $k_{\mathrm{on}} L_{0}$. Clearly, these conditions can be fulfilled in two ways, namely by $L_{0}$ being large relative to $k_{\text {out }}, k_{\mathrm{off}}$ and $k_{\mathrm{e}(\mathrm{L})}$ (as we have already considered) or by $k_{\mathrm{on}}$ being large relative to $k_{\mathrm{out}}, k_{\mathrm{off}}$ and $k_{\mathrm{e}(\mathrm{L})}$ (with no corresponding requirement that $L_{0}$ also be large). In this second case, the results given by (19) and (20) also hold. We note that even for a large (fixed) value of $L_{0}$, if $K_{\mathrm{D}}$ becomes sufficiently large, then $k_{\mathrm{on}} L_{0}$ will no longer be large relative to $k_{\text {off }}$ and so this approximation fails.

\section{Validation and Further Analysis of the Approximations}

We now compare these predicted approximate values with numerical values obtained from the differential equations. We use the values of the constants from the omalizumab case study, as given in Table 1. Since $\mu$ is quite small, the second approximation will be valid in this case. Solving the differential equations numerically gives the true minimum of $R$ to be $R_{\min }=0.5811$. Our two approximations give values of

$$
R_{\min }^{(1)}=0.5203, \quad R_{\min }^{(2)}=0.5787,
$$

from which we can see that the second approximation gives a better result

It is also interesting to consider these approximations over a range of values of $K_{\mathrm{D}}=k_{\mathrm{off}} / k_{\mathrm{on}}$. In the omalizumab study we have the parameter values $k_{\text {off }}=0.9$ and $k_{\text {on }}=0.592$, hence $K_{\mathrm{D}}=1.52$. To make it easier to compare results for varying either $k_{\mathrm{on}}$ or $k_{\mathrm{off}}$, we first fix $k_{\mathrm{on}}=0.592$ and vary $k_{\mathrm{off}}$ via $k_{\mathrm{off}}=0.592 K_{\mathrm{D}}$ and then we fix $k_{\mathrm{off}}=0.9$ and vary $k_{\mathrm{on}}=0.9 / K_{\mathrm{D}}$. The resulting $R_{\min }$ data points from the numerical simulation of the differential equations and approximation curves $R_{\mathrm{min}}^{(1)}$ and $R_{\mathrm{min}}^{(2)}$ are plotted as functions of the variable $K_{\mathrm{D}}$ in Fig. 5 . This illustrates that $R_{\min }^{(1)}$ is a good approximation for all values of $K_{\mathrm{D}}$. For small values of $K_{\mathrm{D}}$, the approximation $R_{\text {min }}^{(2)}$ is better than $R_{\min }^{(1)}$, but for larger values of $K_{\mathrm{D}}$, the approximation $R_{\text {min }}^{(2)}$ starts to fail as expected. For $k_{\text {off }}=0$ and $k_{\text {on }}=0.592$, we have the approximation $R_{\min }^{(1)}=R_{\min }^{(2)}=0.2765$, while the numerical simulation gives $R_{\min }=0.3014$. For $k_{\text {off }}=0.9$ and $k_{\text {on }} \rightarrow \infty$, we have both the simulation and the approximations converging to zero.

It can be seen that the approximation $R_{\min }^{(1)}$ corresponds well to the results obtained by simulation over the whole range of $K_{\mathrm{D}}$ values, while the approximation $R_{\mathrm{min}}^{(2)}$ is a good approximation 
(a)

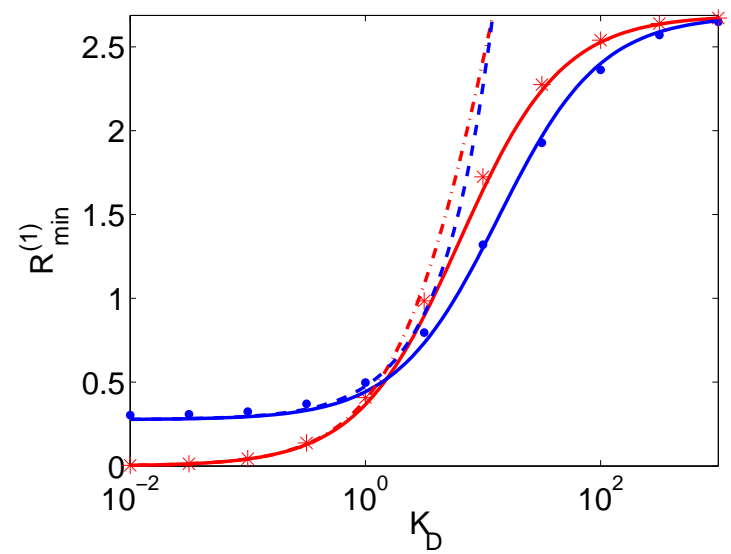

(b)

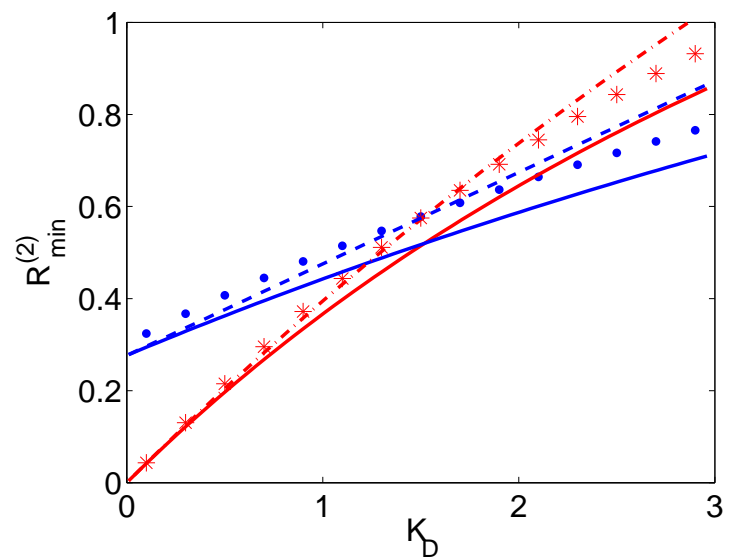

Figure 5: The minimal value of $R$ as a function of $K_{\mathrm{D}}$ with (a) $K_{\mathrm{D}}$ plotted on a log scale for a wide range of $K_{\mathrm{D}}$ values and (b) $K_{\mathrm{D}}$ plotted on a linear scale for $K_{\mathrm{D}}$ small. In both plots, the red solid curve $\left(R_{\text {min }}^{(1)}\right)$ and dash-dotted curve $\left(R_{\min }^{(2)}\right)$ and points $*$ (numerical simulation) are for $k_{\mathrm{off}}=0.9$ and $k_{\mathrm{on}}=0.9 / K_{\mathrm{D}}$. Similarly, the blue solid curve $\left(R_{\min }^{(1)}\right)$ and dashed curve $\left(R_{\min }^{(2)}\right)$ and points $\bullet$ (numerical simulation) are for $k_{\mathrm{on}}=0.592$ and $k_{\text {off }}=0.592 K_{\mathrm{D}}$

when $R_{\min } / R_{0}$ is small, which occurs when either $L_{0}$ or $k_{\text {on }}$ is large. Thus the analytical expressions capture the relationship between the potency $\left(R_{\min }\right)$ and the affinity constants $\left(k_{\text {off }}\right.$ and $k_{\text {on }}$ ) very well.

Another way to view the expression for $R_{\min }^{(1)}$ is by plotting it as a function of the two variables $\log _{10}\left(k_{\mathrm{off}}\right)$ and $\log _{10}\left(k_{\mathrm{on}}\right)$ as shown in Fig. 6. The $R_{\min }^{(1)}$ contours of this surface are shown in Fig. 7 , while the contours with $k_{\text {off }}$ and $k_{\text {on }}$ constant are shown in Figs 8 and 9 respectively. We note in Fig. 7 that when $\log _{10}\left(k_{\text {off }}\right)$ is positive, the contours of $R_{\min }^{(1)}$ appear to be approximately straight lines of slope one. On the other hand, when $\log _{10}\left(k_{\text {off }}\right)$ is less than -1 , the contours are approximately horizontal lines. This can be explained as follows.

If $k_{\text {off }} \gg k_{\text {out }}$, then $k_{\text {off }}$ will make a negligible contribution to the numerator and the denominator of $R_{\min }^{(1)}$ and so we can set it to zero, giving

$$
R_{\text {min }}^{(1)} \approx \frac{R_{0} k_{\text {off }}}{k_{\text {on }}\left(L_{0}-R_{0}\right)+k_{\text {off }}}=\frac{R_{0} K_{\mathrm{D}}}{L_{0}-R_{0}+K_{\mathrm{D}}}, \quad \text { for } k_{\text {off }} \gg k_{\text {out }} .
$$

Thus, in this case, $R_{\text {min }}^{(1)}$ depends only on the single parameter $K_{\mathrm{D}}=k_{\text {off }} / k_{\text {on }}$ and not separately on $k_{\text {off }}$ and $k_{\text {on }}$. If we now consider a contour which corresponds to a constant value of $R_{\min }=R_{c}$, for some given constant $R_{c}$, then we can solve the equation $R_{\min }^{(1)}=R_{c}$ for $K_{\mathrm{D}}$ giving

$$
K_{\mathrm{D}}=\frac{R_{c}\left(L_{0}-R_{0}\right)}{R_{0}-R_{c}}, \quad \text { for } k_{\text {off }} \gg k_{\text {out }} .
$$

Taking logs, we then find that

$$
\log _{10}\left(k_{\text {on }}\right)=\log _{10}\left(k_{\text {off }}\right)-\log \left(\frac{R_{c}\left(L_{0}-R_{0}\right)}{R_{0}-R_{c}}\right), \quad \text { for } k_{\text {off }} \gg k_{\text {out }}
$$

and so the relationship between $\log _{10}\left(k_{\text {off }}\right)$ and $\log _{10}\left(k_{\text {on }}\right)$ is linear with slope one, as seen in Fig. 7 , for $k_{\text {off }}$ sufficiently large.

At the other extreme, when $k_{\text {off }} \ll k_{\text {out }}$, then $k_{\text {off }}$ will be negligible compared to $k_{\text {out }}$ and so, setting it to zero, we obtain

$$
R_{\text {min }}^{(1)} \approx \frac{R_{0} k_{\text {out }}}{k_{\text {on }}\left(L_{0}-R_{0}\right)+k_{\text {out }}}, \quad \text { for } k_{\text {off }} \ll k_{\text {out }} .
$$

In this case, setting $R_{\min }^{(1)}=R_{c}$ and solving for $k_{\mathrm{on}}$, we obtain

$$
k_{\mathrm{on}}=\frac{k_{\mathrm{out}}\left(R_{0}-R_{c}\right)}{R_{c}\left(L_{0}-R_{0}\right)}, \quad \text { for } k_{\mathrm{off}} \ll k_{\text {out }}
$$




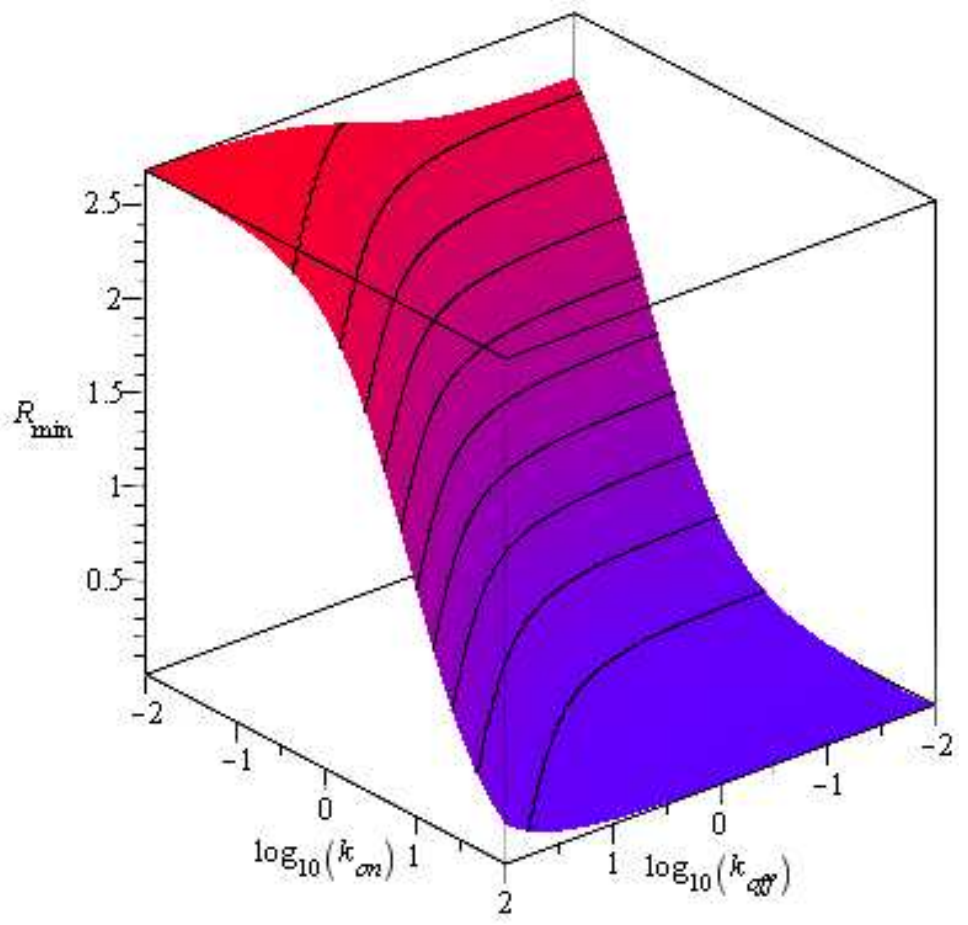

Figure 6: Plot of $R_{\min }^{(1)}$ given by (14) as a function of $\log _{10}\left(k_{\mathrm{off}}\right)$ and $\log _{10}\left(k_{\mathrm{on}}\right)$.

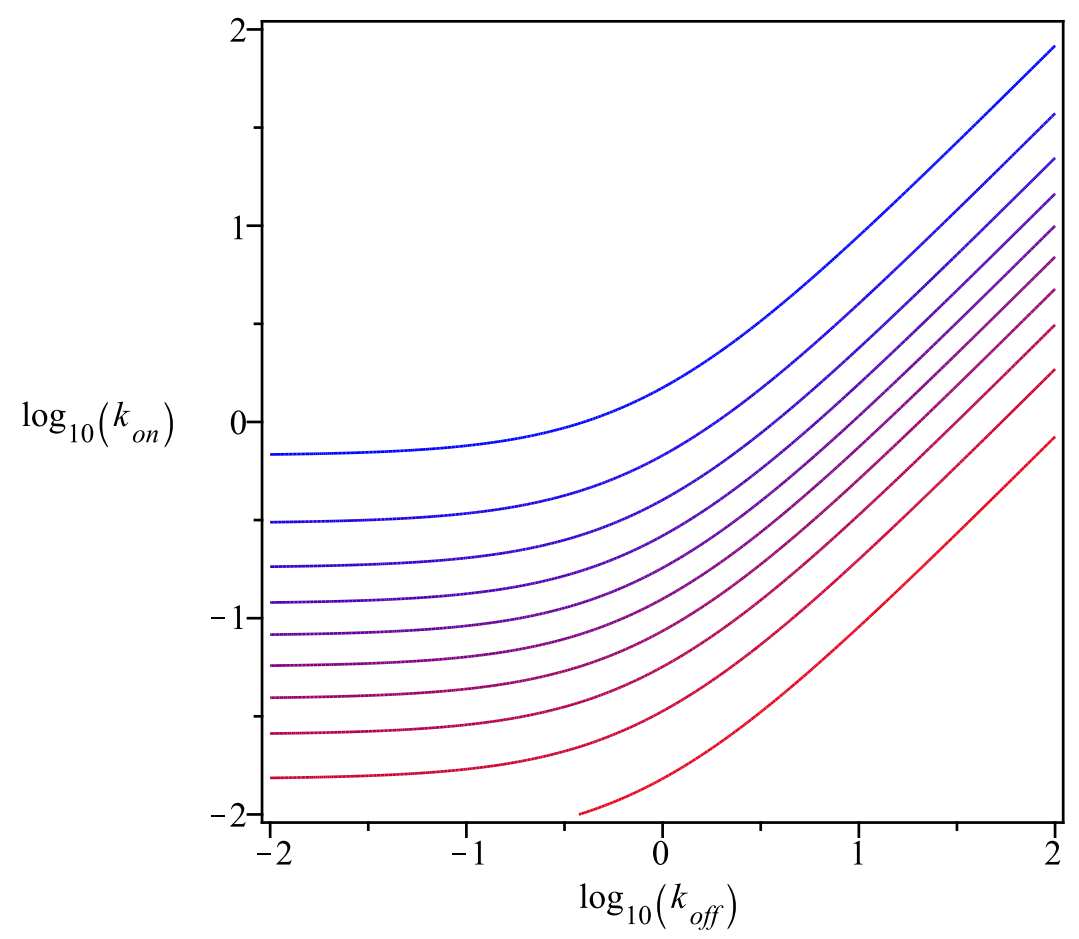

Figure 7: Contours of the surface shown in Fig. 6 for constant values of $R_{\min }$. 


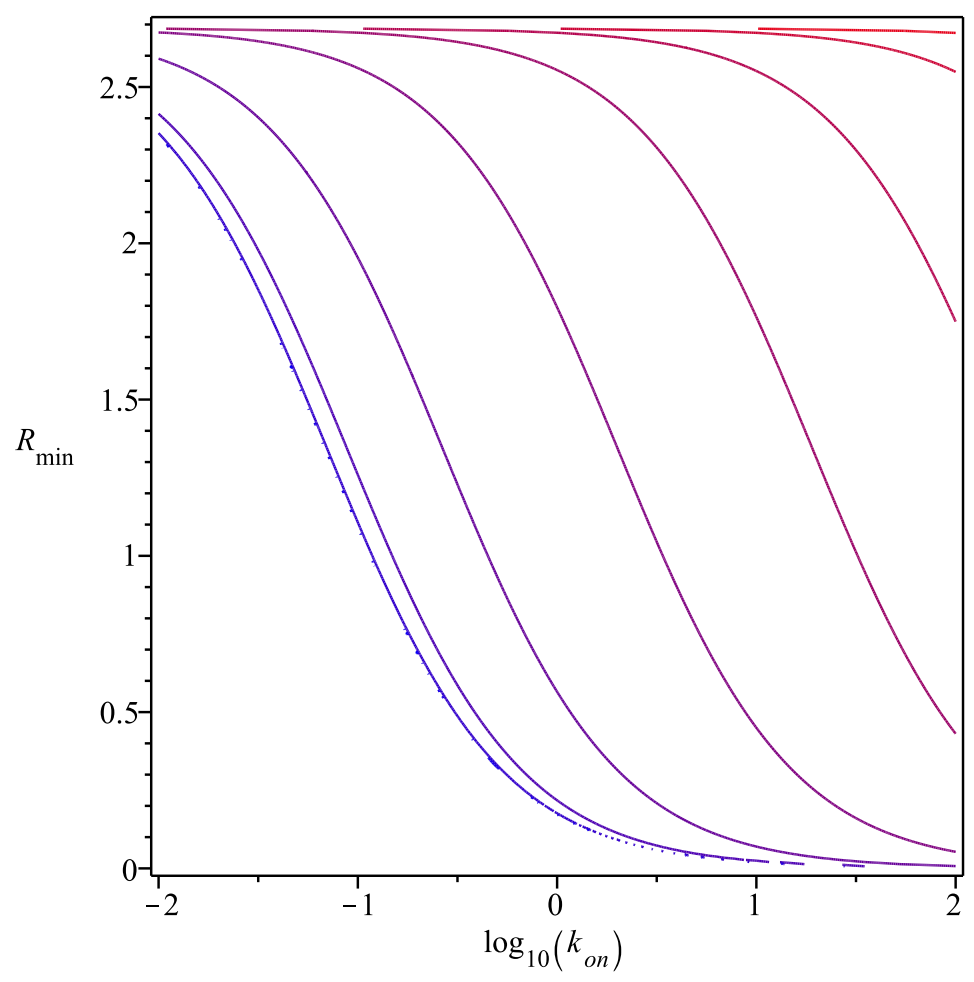

Figure 8: Contours of the surface shown in Fig. 6 for constant values of $k_{\text {off }}$.

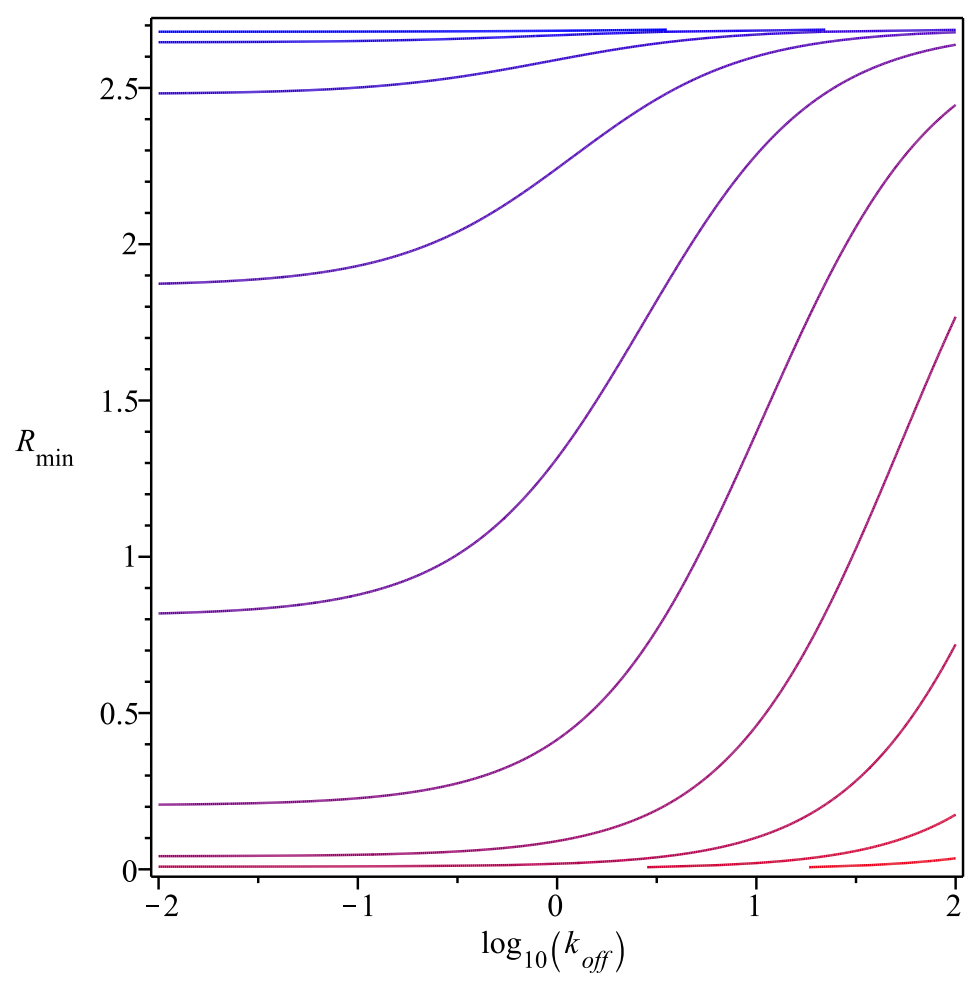

Figure 9: Contours of the surface shown in Fig. 6 for constant values of $k_{\mathrm{on}}$. 
and clearly this expression for $k_{\text {on }}$ does not depend on $k_{\text {off }}$ (since we set it to zero!) and so $\log _{10}\left(k_{\text {on }}\right)$ is a constant.

Thus, the contours asymptote to a constant as $\log _{10}\left(k_{\text {off }}\right) \rightarrow-\infty\left(k_{\text {off }} \rightarrow 0\right)$ and asymptote to a straight line of slope one as $\log _{10}\left(k_{\text {off }}\right) \rightarrow \infty\left(k_{\text {off }} \rightarrow \infty\right)$. The in-between region, when $k_{\text {off }}$ and $k_{\text {out }}$ are of similar order, gives the curve that joins these two straight lines.

We are now able to derive further information on the effect of varying $k_{\text {off }}$ or $k_{\text {on }}$ on $R_{\min }$. When $k_{\text {off }}$ is large relative to $k_{\text {out }}$, we have seen that $R_{\text {min }}^{(1)}$ effectively depends only on the single parameter $K_{\mathrm{D}}$, in which case increasing $k_{\text {on }}$ by a factor of $\alpha$ will have the same effect on $R_{\min }$ as decreasing $k_{\text {off }}$ by a factor of $\alpha$. However, when $k_{\text {off }}$ is either small or of similar magnitude to $k_{\text {out }}$ (as is the case for omalizumab), then increasing $k_{\text {on }}$ by a factor of $\alpha$ will have a much greater effect than reducing $k_{\text {off }}$ by a factor of $\alpha$.

We note that over the whole range of values of $\log _{10}\left(k_{\text {off }}\right)$ to move from one contour in Fig. 7 to a lower one can always be achieved by a relatively small increase in $\log _{10}\left(k_{\text {on }}\right)$. However, moving to a lower contour by decreasing $\log _{10}\left(k_{\text {off }}\right)$ can easily be achieved with a relatively small decrease when $\log _{10}\left(k_{\text {off }}\right)$ is large, but requires a much larger decrease for smaller values of $\log _{10}\left(k_{\text {off }}\right)$, and for smaller values still, it is not possible to move to a lower contour by decreasing $\log _{10}\left(k_{\text {off }}\right)$. This is consistent with the results shown in Fig. 5.

Finally, we consider what increase in $k_{\text {on }}$, or decrease in $k_{\text {off }}$, is required to reduce $R_{\min }$ by a factor of two (which correponds to increasing the efficacy from say $90 \%$ to $95 \%$ ). We suppose that $R_{\text {min }}^{(1)}=R_{c}$ when $k_{\mathrm{on}}=k_{\mathrm{on}}^{0}$ and that $R_{\min }^{(1)}=R_{c} / 2$ when $k_{\mathrm{on}}=k_{\mathrm{on}}^{1}$. This gives the two equations

$$
R_{c}=\frac{R_{0}\left(k_{\text {off }}+k_{\text {out }}\right)}{k_{\text {on }}^{0}\left(L_{0}-R_{0}\right)+k_{\text {off }}+k_{\text {out }}}, \quad \frac{R_{c}}{2}=\frac{R_{0}\left(k_{\text {off }}+k_{\text {out }}\right)}{k_{\text {on }}^{1}\left(L_{0}-R_{0}\right)+k_{\text {off }}+k_{\text {out }}} .
$$

Eliminating $R_{c}$ from these equations and solving for $k_{\text {on }}^{1}$ gives

$$
k_{\mathrm{on}}^{1}=2 k_{\mathrm{on}}^{0}+\frac{k_{\mathrm{off}}+k_{\mathrm{out}}}{L_{0}-R_{0}},
$$

and so we conclude that to reduce $R_{\min }$ by a factor of two, $k_{\text {on }}$ has to be more than doubled, since the second term on the right of $(21)$ is positive.

If we define $K_{\mathrm{D}}^{0}=k_{\mathrm{off}} / k_{\mathrm{on}}^{0}$ and $K_{\mathrm{D}}^{1}=k_{\mathrm{off}} / k_{\mathrm{on}}^{1}$, then from (21) we find that

$$
K_{\mathrm{D}}^{1}=\frac{1}{2} K_{\mathrm{D}}^{0}\left(\frac{2 k_{\mathrm{on}}^{0}\left(L_{0}-R_{0}\right)}{2 k_{\mathrm{on}}^{0}\left(L_{0}-R_{0}\right)+k_{\mathrm{off}}+k_{\mathrm{out}}}\right) .
$$

Since the term in the brackets is less than one, clearly $K_{\mathrm{D}}$ must be reduced by a factor greater than two in order to reduce $R_{\text {min }}$ by a factor or two, which is consistent with the above statement regarding $k_{\mathrm{on}}$.

A similar calculation where $k_{\text {off }}$ is varied rather than $k_{\text {on }}$ gives

$$
k_{\text {off }}^{1}=\frac{1}{2} k_{\text {off }}^{0}-\frac{1}{2} k_{\text {out }}-\frac{\left(k_{\text {off }}^{0}+k_{\text {out }}\right)^{2}}{4 k_{\text {on }}\left(L_{0}-R_{0}\right)+2\left(k_{\text {off }}^{0}+k_{\text {out }}\right)} .
$$

In this case, to reduce $R_{\text {min }}$ by a factor of two, $k_{\text {off }}$ must be reduced by more than a factor of two. Moreover, it is quite possible that this formula gives $k_{\text {off }}^{1}<0$, which of course implies in such a case that it is not possible to reduce $R_{\text {min }}$ by a factor of two by reducing $k_{\text {off }}$, which we have already noted above.

If we define $\tilde{K}_{D}^{0}=k_{\text {off }}^{0} / k_{\text {on }}$ and $\tilde{K}_{D}^{1}=k_{\text {off }}^{1} / k_{\text {on }}$, then from $(22)$ we have that

$$
\begin{aligned}
\tilde{K}_{D}^{1} & =\frac{1}{2} \tilde{K}_{D}^{0}-\frac{k_{\text {out }}}{2 k_{\text {on }}}-\frac{\left(k_{\text {off }}^{0}+k_{\text {out }}\right)^{2}}{k_{\text {on }}\left[4 k_{\text {on }}\left(L_{0}-R_{0}\right)+2\left(k_{\text {off }}^{0}+k_{\text {out }}\right)\right]} \\
& =\frac{1}{2} \tilde{K}_{D}^{0}-\frac{k}{2}-\frac{\left(\tilde{K}_{D}^{0}+k\right)^{2}}{4\left(L_{0}-R_{0}\right)+2\left(\tilde{K}_{D}^{0}+k\right)}
\end{aligned}
$$

where $k=k_{\text {out }} / k_{\text {on }}$. The comments above regarding $k_{\text {off }}$ apply also to $\tilde{K}_{D}$. 


\section{Discussion and Conclusion}

The main objective of the work presented in this paper was to explore the TMDD model through a rigorous mathematical analysis with regards to the relationship between the target affinity of a mAb versus its in vivo potency. In our experience, this topic invariably gets raised in $\mathrm{mAb}$ drug discovery and development programs, mainly because the maximum dose for routine clinical use in patients is typically more stringently constrained for mAbs compared to small molecules due to non-oral route of administration, formulation complexities and cost of goods, and because increasing the affinity of a mAb is a time-consuming process.

The first, perhaps obvious, conclusion of our analysis is that for a given dose $\left(L_{0}\right)$ the minimum value of free target $\left(R_{\min }\right)$ can be decreased (equivalent to increasing in vivo potency) by increasing $k_{\text {on }}$ or by decreasing $k_{\text {off }}$. While this conclusion may be obvious, what may not be so apparent is that there is a significant difference in the minimum receptor obtained when $K_{\mathrm{D}}$ is reduced either by reducing $k_{\text {off }}$ or by increasing $k_{\mathrm{on}}$. We note from (15) that as $k_{\mathrm{on}} \rightarrow \infty$, then $R_{\mathrm{min}}^{(1)} \rightarrow 0$, whereas from (16), as $k_{\mathrm{off}} \rightarrow 0$, then $R_{\text {min }}^{(1)}$ tends to a non-zero value, with the same limits being obtained from $R_{\min }^{(2)}$, as already noted. Thus, we have a saturation effect when decreasing $k_{\text {off }}$, in that further reductions in the value of $k_{\text {off }}$ will only yield limited reductions in $R_{\min }$, whereas there is no such saturation when increasing $k_{\mathrm{on}}$, so that $R_{\min }$ is consistently reduced for increasing values of $k_{\mathrm{on}}$.

We see from (16) and (20) that the limiting value of $R_{\min }$ as $k_{\mathrm{off}} \rightarrow 0$ in both cases is given by

$$
\lim _{k_{\text {off }} \rightarrow 0} R_{\min }^{(1)}=\lim _{k_{\text {off }} \rightarrow 0} R_{\min }^{(2)}=\frac{k_{\text {in }} k_{\text {out }}}{k_{\text {on }}\left(k_{\text {out }} L_{0}-k_{\text {in }}\right)+k_{\text {out }}^{2}},
$$

where we have substituted $R_{0}=k_{\text {in }} / k_{\text {out }}$. Thus, we can see that this saturation level will be reduced by increasing $k_{\text {on }}$ or $L_{0}$. Theoretically, the saturation level could also be reduced by decreasing $k_{\text {in }}$ or by increasing $k_{\text {out }}$.

This sheds new light on our previous work [2] in which, through simulations, we predicted that the maximum increase in potency that could be achieved with an anti-IgE antibody by only reducing the $k_{\text {off }}$ would be approximately two-fold compared to omalizumab, and that this would be achieved with a five-to-ten-fold increase in affinity. Clinical data reported subsequently by Putnam et al. [4] on the high-affinity anti-IgE mAb, HAE1, were consistent with this prediction, since it was shown that HAE1 achieved an approximately two-fold improvement in in vivo potency compared to omalizumab, whereas it displayed a more than twenty-fold higher affinity for IgE. Interestingly, the data presented in [4] show that the affinity improvement of HAE1 compared to omalizumab was entirely driven by a reduction in $k_{\text {off }}$, consistent with our prediction that this parameter is associated with a saturation effect regarding its impact on in vivo potency.

As far as we are aware there is no experimental data to support our hypothesis that such a saturation effect does not exist for $k_{\text {on }}$, but our analysis does suggest that a mAb potency optimisation strategy focused on increasing $k_{\text {on }}$ rather than decreasing $k_{\text {off }}$ could be advantageous. Currently, the majority of marketed mAbs and those in clinical development are IgE's of about $150 \mathrm{kDa}$ size and their $k_{\mathrm{on}}$ values are generally uniform and limited by their size. However, even if rational optimisation of $k_{\text {on }}$ is currently not experimentally possible, our analysis indicates that between two antibodies of sufficiently low $k_{\text {off }}$ (in the saturation effect range), one antibody with a higher $k_{\text {on }}$ is more potent even if it may have higher $k_{\text {off }}$ and hence, potentially higher overall $K_{\mathrm{D}}$. While this conclusion may already have been derived in an empirical manner within certain areas of biologics discovery (especially with the extensive research into highly labile targets such as interleukins) we have provided the first systematic quantitative analysis that can guide rational optimisation of mAbs within the context of the TMDD framework. For example, in recent years, significant efforts have been put into the development of novel human and non-human scaffolds ('nanobodies') of much smaller size (see [17, 18]). Although currently these efforts appear to be mainly motivated by predicted improvement of tissue penetration, systemic stability and preferential cleft recognition [19], an intriguing question that follows on from the present analysis 
is whether nanobodies could display faster $k_{\text {on }}$ rates due to their smaller size and therefore may be more amenable to optimisation of in vivo potency compared to traditional mAbs.

It should be pointed out that an important assumption in our analysis is that $L_{0}$ must be significantly larger than $R_{0}$. Of course this is not a rigorous statement, but the important criterion is that the parameter $\mu=R_{0} / L_{0}$ must be small, as was assumed in the mathematical analysis. We have shown that the value of $\mu=0.18144$ for the $\operatorname{IgE~mAb}$ omalizumab is sufficiently small for the analysis to work well. However, while this assumption may generally hold true for blocking and neutralising antibodies, it may not be true for agonists and in cases where pharmacological effect may be exerted at low levels of target occupancy. Therefore, our conclusions may not be valid in those cases. However, we do not believe this greatly limits applicability of the simplification we have provided, since in the cases where the underlying assumption is violated, lower doses are only required for clinical efficacy and therefore affinity requirements tend to be less stringent. It should also be noted that this analysis is only applicable for a rather simple antigen-antibody system which interacts without diffusion barriers, avidity effects, and other complexities. These complicating factors should be considered to be able to extend the model to more realistic drug discovery situations.

In conclusion, our analysis of the TMDD model has provided a mathematical framework that relates intrinsic pharmacological and pharmacokinetic properties of mAbs to their in vivo potency. The finding that the greatest potency improvements can be achieved, at least in theory, through modulation of $k_{\text {on }}$ could provide a basis for new strategies to drive the discovery of a new generation of mAbs. The simple formula we have provided can also substantially reduce the need for complex PKPD analyses resources to get an initial estimate of required affinity at the lead optimisation stage.

\section{Acknowledgements}

This work was financially supported through the Biopharma Skills Project of the Universities of Surrey and Reading, jointly funded by the Higher Education Funding Council for England's Economic Challenge Investment Fund (ECIF) and the South East England Development Agency (SEEDA).

We are grateful to Prof Bert Peletier (Leiden) for helpful discussions and encouragement. We also thank Dr David Lloyd (Surrey) for his interest and advice.

\section{References}

[1] A.L. Nelson, E. Dhimolea, J.M. Reichert, Development trends for human monoclonal antibody therapeutics, Nat. Rev. Drug Discov. 9 (2010) 767-774.

[2] B.M. Agoram, S.W. Martin, P.H. van der Graaf, The role of mechanism-based pharmacokinetic-pharmacodynamic (PK-PD) modelling in translational research of biologics, Drug Discov. Today 12 (2007) 1018-1024.

[3] B.M. Agoram, Use of pharmacokinetic/pharmacodynamic modelling for starting dose selection in first-in-human trials of high-risk biologics, Br. J. Clin. Pharmacol. 67 (2009) 153-160.

[4] W.S. Putnam, J. Li, J. Haggstrom, C. Ng, S. Kadkhodayan-Fischer, M. Cheu, Y. Deniz, H. Lowman, P. Fielder, J. Visich, A. Joshi, N.S. Jumbe, Use of quantitative pharmacology in the development of HAE1, a high-affinity anti-IgE monoclonal antibody, AAPS J. 10 (2008) 425-430.

[5] M. Tabrizi, C. Funelas, H. Suria, Application of quantitative pharmacology in development of therapeutic monoclonal antibodies, AAPS J. 12 (2010) 592-601. 
[6] G. Levy, Pharmacologic target-mediated drug disposition, Clin. Pharmacol. Ther. 56 (1994) $248-52$.

[7] D.E. Mager, W.J. Jusko, General pharmacokinetic model for drugs exhibiting target-mediated drug disposition, J. Pharmacokinet. Pharmacodyn. 28 (2001) 507-32.

[8] D.E. Mager, Target-mediated drug disposition and dynamics, Biochem. Pharmacol. 72 (2006) $1-10$.

[9] L. Gibiansky, E. Gibiansky, Target-mediated drug disposition model: approximations, identifiability of model parameters and applications to the population pharmacokineticpharmacodynamic modeling of biologics, Expert Opin. Drug Metab. Toxicol. 5 (2009) 803-812.

[10] L.A. Peletier, J. Gabrielsson, Dynamics of target-mediated drug disposition, Eur. J. Pharm. Sci. 38 (2009) 445-464.

[11] J.P. Davda, R.J. Hansen, Properties of a general PK/PD model of antibody-ligand interactions for therapeutic antibodies that bind to soluble endogenous targets, MAbs 2 (2010) 576-588.

[12] A.K. Abraham, L. Kagan, S. Kumar, D.E. Mager, Type I interferon receptor is a primary regulator of target-mediated drug disposition of interferon-beta in mice, J. Pharmacol. Exp. Ther. 334 (2010) 327-332.

[13] B.F. Krippendorff, K. Kuester, C. Kloft, W. Huisinga, Nonlinear pharmacokinetics of therapeutic proteins resulting from receptor mediated endocytosis, J. Pharmacokinet. Pharmacodyn. 36 (2009) 239-260.

[14] N. Hayashi, Y. Tsukamoto, W.M. Sallas, P.J. Lowe, A mechanism-based binding model for the population pharmacokinetics and pharmacodynamics of omalizumab, Br. J. Clin. Pharmacol. 63 (2007) 548-561.

[15] C.A. Sarkar, D.A. Lauffenburger, Cell-level pharmacokinetic model of granulocyte colonystimulating factor: implications for ligand lifetime and potency in vivo, Mol. Pharmacol. 63 (2003) 147-158.

[16] D.E. Mager, W. Krzyzanski, Quasi-equilibrium pharmacokinetic model for drugs exhibiting target-mediated drug disposition, Pharm. Res. 22 (2005) 1589-1596.

[17] D.S. Dimitrov, Engineered $\mathrm{CH}_{2}$ domains (nanoantibodies), MAbs 1 (2009) 26-28.

[18] F. van Bockstaele, J.B. Holz, H. Revets, The development of nanobodies for therapeutic applications, Curr. Opin. Investig. Drugs 10 (2009) 1212-1224.

[19] E. de Genst, K. Silence, K. Decanniere, K. Conrath, R. Loris, J. Kinne, S. Muyldermans, L. Wyns, Molecular basis for the preferential cleft recognition by dromedary heavy-chain antibodies, Proc. Natl. Acad. Sci. US A 103 (2006) 4586-4591. 